\title{
Entre lagunas y el mar: uso del espacio por cazadores-recolectores en un paisaje lagunar de la costa norte de Santa Cruz
}

\author{
(4) Heidi Hammond" y Leandro Zilio**
}

Recibido: 17 de febrero de 2017

Aceptado:

21 de junio de 2017

\section{Resumen}

Se presentan los resultados del estudio arqueológico de un sector pericostero ubicado a $20 \mathrm{~km}$ al norte de la ciudad de Puerto Deseado, en el área de la costa norte de Santa Cruz. El tramo analizado se caracteriza por la presencia de ocho cuerpos lagunares endorreicos con contenido ocasional de agua aportado por las lluvias y la escorrentía superficial. El objetivo de este trabajo es evaluar la presencia, distribución espacial y las características del registro arqueológico en este paisaje lagunar, adyacente a una franja de litoral atlántico definida en estudios previos por la ausencia de materiales arqueológicos. Se realizaron prospecciones sistemáticas en el campo y en los márgenes de la totalidad de las lagunas se identificaron materiales arqueológicos. Además se llevaron a cabo dos sondeos estratigráficos y una datación radiocarbónica. Los resultados obtenidos se discuten en el marco de las evidencias disponibles para el área de estudio. Finalmente, se plantea que las poblaciones pasadas habrían hecho un uso diferencial, aunque complementario, del espacio lagunar y el litoral marítimo adyacente. La complementariedad en el uso de los espacios habría estado relacionada con la estructura de los recursos y las características de los sustratos de ocupación disponibles.

\section{Between lagoons and the sea: use of space by hunter-gatherers of a lagoon landscape on the Northern Coast of Santa Cruz}

\footnotetext{
Abstract

Here we present the results of an archaeological study of a peri-coastal sector located 20 $\mathrm{km}$ North of the city of Puerto Deseado, Northern Coast of Santa Cruz. The analyzed area is characterized by the presence of eight endorheic lagoons, which occasionally accumulate water due to rainfall and the surface runoff. The main aim of this paper is to assess the archaeological presence, its spatial distribution and its characteristics within this lagoon landscape. This landscape is adjacent to a strip of Atlantic coastline defined in previous studies by the absence of archaeological material. Systematic surveys were

Palabras claves

Paisaje lagunar Litoral atlántico Cazadores-recolectores Uso del espacio Costa norte de Santa Cruz

Keywords

Lagoon landscape Atlantic coast Hunter-gatherers Use of space Northern Coast of Santa Cruz 
carried out in the field. We recovered archaeological material from the shoreline of all these lagoons. In addition, two stratigraphic excavations and radiocarbon dating of remains was undertaken. The results obtained are discussed here within the framework of the available evidence for the study area. Finally, it is argued that past populations would have made differential but complementary use of the lagoon landscape and the adjacent maritime coast. Complementarity in the use of spaces would have been related to resources structures and to the characteristics of the available occupation substrates.

\section{Introducción}

A fines de la década de 1990 se desarrolló una prospección macrorregional dirigida por la Dra. A. Castro en el área de la costa norte de Santa Cruz (CNSC) con el objetivo de reconocer las características superficiales del registro arqueológico, su distribución y densidad, así como los materiales que conformaban los sitios. Se llevaron a cabo estudios espaciales para establecer patrones distribucionales del registro y las relaciones entre éste y el ambiente (Castro et al., 2003). A partir de estas investigaciones se obtuvo una imagen acerca de las características y la distribución de las evidencias arqueológicas en el área. En este marco, la franja de litoral marítimo ubicada entre las localidades de Cabo Blanco y Puerto Deseado, caracterizada por la presencia de amplios intermareales y playas de rodados, fue definida por la ausencia casi total de restos arqueológicos (Castro et al., 2003, p. 78; Figura 1). En contraste con el sector anterior, otros espacios de la CNSC, como por ejemplo aquellos situados al sur de la ría Deseado, se definieron por la presencia de una alta densidad del registro arqueológico representado principalmente por sitios concheros, enterratorios humanos y sitios de actividades múltiples (Castro y Moreno, 2000; Castro, Moreno, Andolfo y Zubimendi, 2001; Castro et al., 2003; Zubimendi, Castro y Moreno, 2004).

El objetivo general de este trabajo es, entonces, discutir las modalidades de uso del espacio por parte de las poblaciones humanas en el pasado en un sector lagunar adyacente a la franja de litoral atlántico ubicada entre Cabo Blanco y Puerto Deseado. Para ello se evaluó la presencia, distribución espacial y las características del registro arqueológico en un sector ubicado a $20 \mathrm{~km}$ al norte de la ciudad de Puerto Deseado (Figura 1), caracterizado por la presencia de ocho cuerpos lagunares hoy en día secos y con contenido de agua ocasional aportado por las lluvias estacionales y la escorrentía superficial. Este sector lagunar no había sido estudiado en trabajos arqueológicos previos por lo cual los resultados aquí presentados constituyen las primeras evidencias obtenidas para este espacio.

A fin de caracterizar el registro arqueológico del sector pericostero se realizaron prospecciones orientadas hacia la detección de sitios arqueológicos en superficie, así como de restos aislados. Además se llevaron a cabo dos sondeos estratigráficos y una datación radiocarbónica sobre carbón vegetal recuperado en uno de ellos.

A partir de la distribución espacial del registro arqueológico se evaluó la distancia mínima de acarreo o transporte hacia los espacios lagunares de recursos con procedencia geográfica conocida, tales como los moluscos y vertebrados marinos y los rodados costeros. Se analizó la representatividad de especies malacológicas que conforman los sitios en superficie y aquellas recuperadas en las excavaciones, así como los restos óseos de vertebrados y los materiales líticos.

\section{El ambiente y las características geológicas}

El clima en el área de estudio es árido a semiárido y ventoso, con escasas precipitaciones, marcadas amplitudes térmicas diarias, fuerte evaporación y vegetación de tipo estepario. 
El paisaje está conformado por mesetas y aportes producto de las ingresiones marinas y es afectado por diferentes procesos geomorfológicos que actúan en su formación, como drenaje y escorrentía superficial, remoción, deflación y acumulación eólica. El sector lagunar analizado se habría configurado durante el último máximo interglacial en el Pleistoceno final hace alrededor de 120.000 años (MIS 5e; Pedoja et al., 2011). Los cuerpos lagunares son endorreicos, sin descarga fluvial hacia el océano, de poca profundidad y de tamaños variables (Figura 1 y Tabla 1). En todos ellos se observó la depositación de un manto o pluma eólica sobre el margen sur-este como consecuencia de la acumulación de sedimentos eólicos finos, influenciada por los vientos predominantes del oeste y el clima árido. Dos de las lagunas actualmente son salinas, las cuales corresponden a cuerpos someros con escaso desarrollo hacia profundidad, que dejan una costra evaporítica superficial originada por precipitación físico-química (Alonso, 2006).

La franja de costa atlántica adyacente al sector estudiado se caracteriza por la presencia de crestas de playa características de costas de progradación conformadas por sedimentos clásticos gravosos con escasos bioclastos. En estas costas, cada pulso de acreción queda representado morfológicamente por un depósito longilíneo levemente elevado, paralelo o subparalelo a la lineación general de la costa (Codignotto, 1997). En los intermareales ubicados sobre el frente atlántico se identificaron extensas plataformas de abrasión rocosas con disponibilidad de moluscos, principalmente de la especie Nacella magellanica (Hammond y Zilio, 2016a).

\section{Materiales y métodos}

Se llevaron a cabo prospecciones en el campo mediante transectas sistemáticas realizadas por dos personas a pie, distanciadas $5 \mathrm{~m}$ entre sí, por lo que cada operador cubría un ancho de $5 \mathrm{~m}$ de muestreo, que es la superficie máxima estimada confiable que puede controlar visualmente una persona (Foley, 1981). Se prospectó la totalidad del perímetro de un total de ocho cuerpos lagunares. Durante las tareas de muestreo se utilizó material cartográfico y fotográfico del área de interés y un GPS (posicionador global) para la localización de las evidencias arqueológicas en el campo.

El procedimiento seguido en las transectas fue la identificación de loci arqueológicos en los márgenes de los cuerpos lagunares y el registro de una serie de aspectos preestablecidos: emplazamiento geomorfológico, morfología, materiales componentes (malacológico, lítico, óseo, etc.), acción de agentes postdepositacionales y condiciones de visibilidad del registro. Los materiales que componen los sitios fueron clasificados de forma cualitativa según categorías de densidad: Densidad Baja, con presencia de materiales muy dispersos; Densidad Media, partes del locus con materiales dispersos y otros con mayor cantidad de restos en contacto entre sí, formando agrupaciones; Densidad Alta, gran cantidad de materiales muy concentrados y en contacto entre sí (Hammond, 2015). Estas categorías se registraron a partir la delimitación de unidades de muestreo de 1 por $1 \mathrm{~m}$ en el sector central de las acumulaciones arqueológicas. La información relevada fue volcada en una planilla adecuada al muestreo y luego procesada en el laboratorio.

A partir de la información espacial se generaron mapas de localización y de densidad de sitios mediante el empleo de Sistemas de Información Geográfica (SIG) utilizando el software ArcMap 10.1. Se trabajó con el estimador de Densidad Kernel, a partir del cual se calcula la densidad de las entidades registradas según la presencia o no de entidades en la vecindad, utilizando el método del “Vecino más cercano" (Moreno Jiménez, 2005).

Se llevaron a cabo dos sondeos de $1 \mathrm{~m}^{2}$ con el fin analizar las características estratigráficas de los sitios. Se realizó el reconocimiento anatómico y taxonómico de los exoesqueletos 


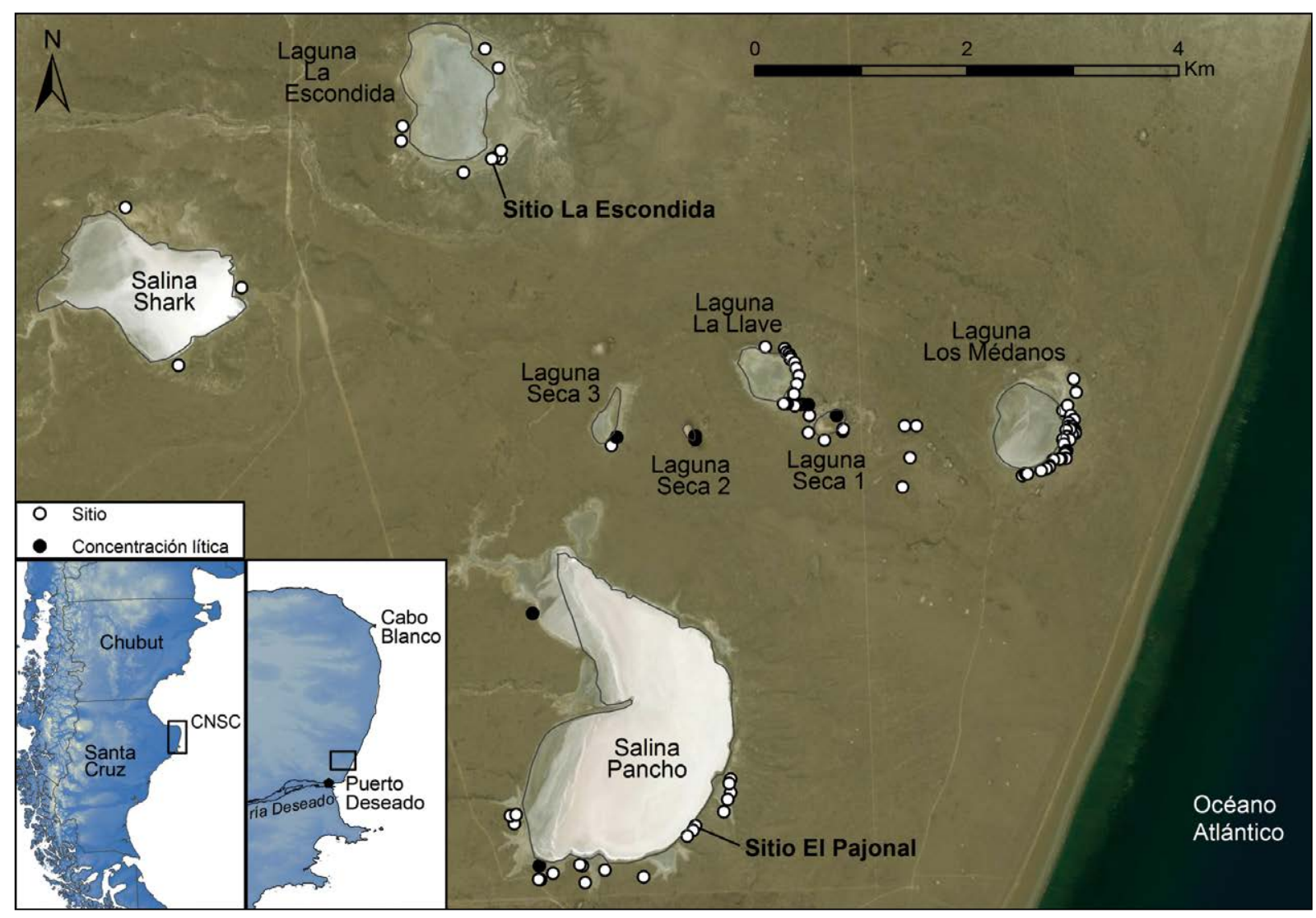

Figura 1. Área de estudio, paisaje lagunar analizado en este trabajo y sitios arqueológicos identificados.

\begin{tabular}{|l|c|c|c|c|c|c|}
\hline \multirow{2}{*}{ Cuerpo lagunar } & \multirow{2}{*}{$\begin{array}{c}\text { Total } \\
\text { sitios }\end{array}$} & \multicolumn{2}{|c|}{ N de sitios con presencia de } & Distancia al litoral & $\begin{array}{c}\text { Área } \\
\text { marítimo }(\mathbf{k m})\end{array}$ \\
\cline { 3 - 5 } & Moluscos & Óseo & Lítico & (km $)$ \\
\hline Salina Shark & 3 & 3 & 0 & 3 & 6,2 & 0,627 \\
Laguna La Escondida & 13 & 13 & 1 & 12 & 4,9 & 0,421 \\
Salina Pancho & 22 & 20 & 9 & 19 & 2,2 & 1,410 \\
Laguna Seca 3 & 2 & 1 & 1 & 2 & 3,6 & 0,029 \\
Laguna Seca 2 & 2 & 0 & 2 & 2 & 3 & 0,004 \\
Laguna Seca 1 & 6 & 5 & 2 & 5 & 2,3 & 0,026 \\
Laguna La Llave & 25 & 24 & 6 & 25 & 2,6 & 0,082 \\
Laguna Los Médanos & 46 & 46 & 7 & 46 & 0,9 & 0,207 \\
\hline Total & $\mathbf{1 1 9}$ & $\mathbf{1 1 2}$ & $\mathbf{2 8}$ & $\mathbf{1 1 4}$ & - & - \\
\hline
\end{tabular}

Tabla 1. Características de los cuerpos lagunares y frecuencia de sitios identificados.

de moluscos y se estableció el número total de restos identificables (NR; total de conchas completas y fragmentos cuantificables; Hammond, 2015). Además se llevó a cabo el análisis biométrico de las conchas de Nacella magellanica para evaluar el tamaño de los restos y discutir posibles variaciones espacio temporales en el marco de la información de este tipo con la que se cuenta para el área de estudio (Hammond y Zilio, 2016b). Nacella magellanica corresponde a la única especie con valor alimenticio de la cual se recuperaron exoesqueletos completos en los sondeos. 
Se identificaron los especímenes óseos determinables a nivel taxonómico de especie cuando fue posible (clase, familia e indeterminados cuando no se alcanzó el nivel específico), se establecieron las partes esqueletales a través de la localización de rasgos diagnósticos y se estableció el NISP (Lyman, 1994).

El estudio de los conjuntos artefactuales líticos y la clasificación de las piezas se realizó a partir de la consideración de variables macroscópicas tecno-morfológicas (Aschero, 1975) y de variables funcionales (Castro, 1994). Los grupos tipológicos considerados en los análisis son: instrumentos formatizados, formas base potenciales, desechos de talla y núcleos. Entre los instrumentos se incluyen los artefactos que presentan filos, puntas o superficies con rastros complementarios. Las formas base potenciales corresponden a todos aquellos artefactos que pueden ser utilizados directamente, es decir, que poseen las características de tamaño que permiten que sean tomados con la mano y filos adecuados para realizar una función aún sin estar formatizados; como también aquellas formas que previo trabajo de talla, retalla y formatización pueden ser transformadas en un instrumento. Dentro de este grupo se consignan lascas y láminas (u hojas según Aschero, 1975) que cumplen con los requisitos antes mencionados. La categoría desechos de talla incluye los subgrupos: microlascas (menores a $5 \mathrm{~mm}^{2}$ ), lascas chicas (entre $10 \mathrm{~mm}^{2}$ y $5 \mathrm{~mm}^{2}$ ), esquirlas (entre $1 \mathrm{~mm}^{2}$ y $10 \mathrm{~mm}^{2}$ ) y desechos propiamente dichos (mayores a $10 \mathrm{~mm}^{2}$ ). Los núcleos se definen como todo nódulo del que se han extraído lascas o láminas (Aschero, 1975, p. 9).

El fechado radiocarbónico se realizó en el Laboratorio de Radiocarbono (CIG-LATYR) y se calibró mediante el software OxCal 4.2 (Bronk Ramsey y Lee, 2013). La calibración se realizó en años AP con dos desvíos estándar, empleando la curva de calibración para el Hemisferio Sur (SHCal13; Hogg et al., 2013).

\section{Resultados}

\section{Análisis espacial}

Se registraron un total de 123 sitios, de los cuales 119 se encuentran en las márgenes de los cuerpos lagunares y cuatro se distribuyen entre lagunas (Tabla 1 y Figuras 1 y 2). Todos ellos corresponden a acumulaciones con morfología plano extendida, conformadas principalmente por exoesqueletos calcáreos de moluscos de la especie Nacella magellanica (también se registraron escasos restos de Aulacomya atra, Adelomelon sp. y Nacella mytilina), restos óseos faunísticos y artefactos líticos. Se registraron además siete concentraciones conformadas únicamente por material lítico disperso (Figura 1).

En un total de 28 sitios se reconoció la presencia de material óseo faunístico (Tabla 1). En general estos restos se encuentran muy fragmentados y con estadios de meteorización elevados. En el campo fue posible identificar restos de pinnípedos, guanacos y aves marinas. En cuanto al material lítico, este se encuentra presente en bajas densidades en un total de 114 sitios. Las materias primas que predominan son la calcedonia de diversos colores y los basaltos, disponibles en general en depósitos secundarios (Ambrústolo, 2011). En menor frecuencia se registraron pórfidos locales, tobas y andesitas distribuidas en depósitos secundarios y obsidiana negra de disponibilidad no local en el área de estudio (Ambrústolo, 2011). Se reconoció principalmente desechos de talla y lascas y en menor frecuencia láminas, bifaces, raspadores, raederas, puntas de proyectil, percutores, núcleos y núcleos bipolares.

A partir del relevamiento superficial se observó que la mayor frecuencia de sitios y densidad de materiales que los conforman se encuentra en las márgenes de los cuerpos 


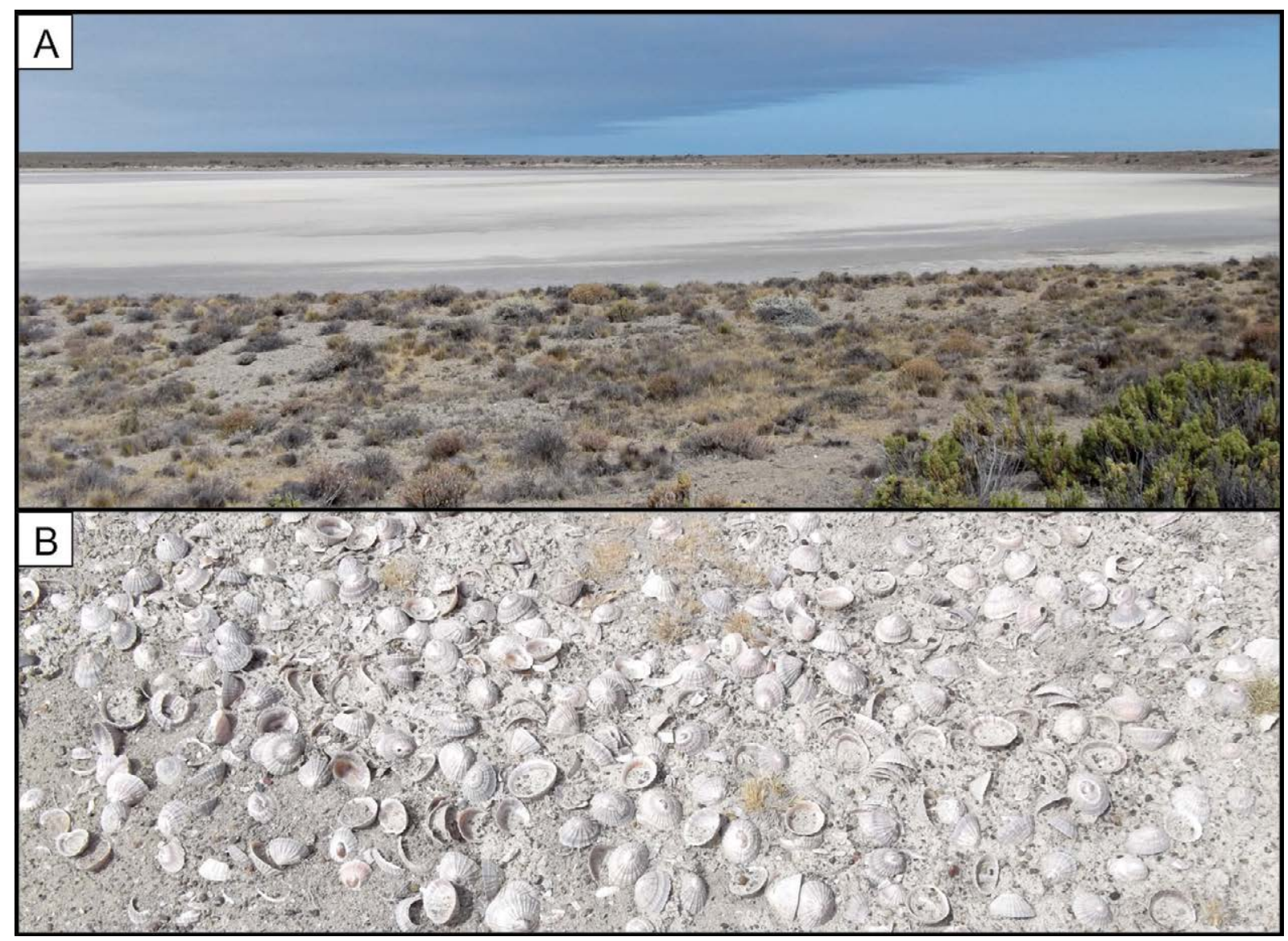

Figura 2. A) Laguna Los Médanos; B) sitio con presencia de exoesqueletos de Nacella magellanica en laguna Los Médanos.

lagunares más cercanos al litoral marítimo y que estas variables decrecen a medida que las lagunas se sitúan a mayor distancia del mar (Figura 3). Esta característica se relaciona con las condiciones de visibilidad de los sitios, ya que aquellos conformados por mayores densidades de materiales (especialmente exoesqueletos de moluscos) presentan mejores condiciones de visibilidad que los sitios conformados por restos arqueológicos dispersos.

En la Figura 3B se representa la densidad de exoesqueletos de moluscos registrados por sitio. Se observa que los sitios con mayor densidad se encuentran principalmente en las márgenes de lagunas cercanas al litoral atlántico y que en estas últimas, a su vez, se registró una mayor cantidad de sitios. El contexto arqueológico con presencia de exoesqueletos de moluscos (Nacella magellanica) más alejado del litoral marítimo se localizó aproximadamente a $7 \mathrm{~km}$.

\section{Excavaciones arqueológicas}

Se realizaron dos sondeos estratigráficos, uno de ellos en el sitio La Escondida (LE), ubicado en la margen sudeste de la laguna homónima; y el otro en el sitio El Pajonal (EP), emplazado en la margen sudeste de la salina Pancho (Figura 1). Estos sitios se encuentran a 4,9 y 2,3 km de la costa del mar, respectivamente. En LE se realizó una datación radiocarbónica a partir de carbón vegetal (Tabla 2).

En ambos sitios los conjuntos arqueomalacológicos están constituidos por restos de Nacella magellanica (Tabla 2). Esta especie ha sido definida como una de las tres principales con alto valor alimenticio para las poblaciones humanas pasadas, junto 


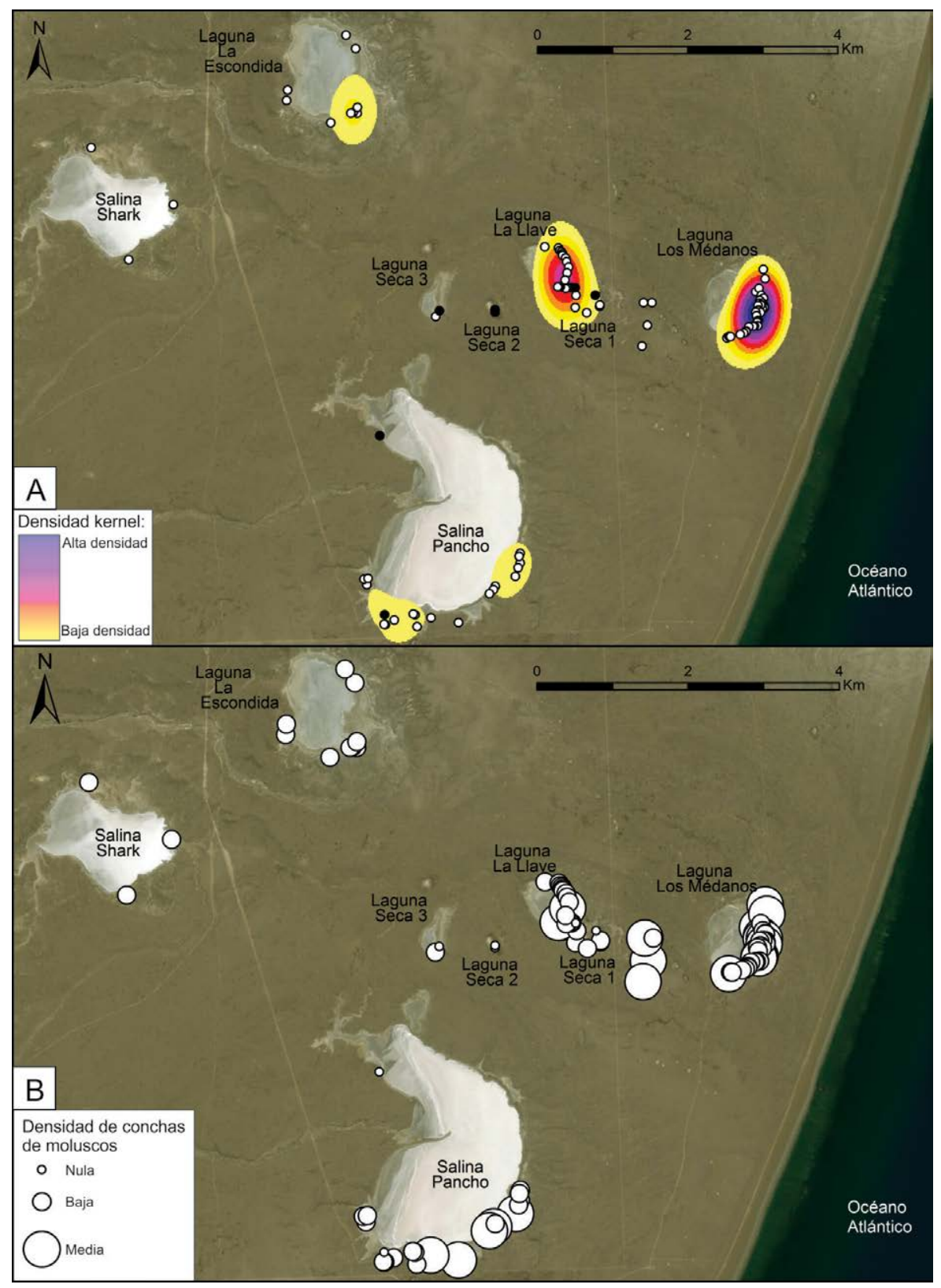

Figura 3. A) Densidad Kernel de sitios en el sector lagunar; B) densidad de exoesqueletos de moluscos registrados en torno a los cuerpos lagunares.

con los mitílidos Aulacomya atra y Mytilus edulis (Zubimendi, 2012; Hammond, 2015; Hammond y Zilio, 2016b). La media y desvío estándar de las dimensiones de las conchas de Nacella magellanica para LE son: largo: 44,6 $\pm 4,6 \mathrm{~mm}$; ancho: $36,6 \pm$ $4 \mathrm{~mm}$ y alto: $19,1 \pm 3,2 \mathrm{~mm}(\mathrm{n}=173)$; para $\mathrm{EP}$, largo: $42,1 \pm 5,1 \mathrm{~mm}$; ancho: $33,8 \pm$ $3,9 \mathrm{~mm}$ y alto: $19,3 \pm 4 \mathrm{~mm}(\mathrm{n}=75)$. En EP se recuperó además un fragmento con presencia del umbo correspondiente a Mytilus edulis.

En la Tabla 2 se presenta el NISP de elementos óseos por taxón; en todos los casos la preservación de las superficies óseas es buena. Los restos de guanaco de LE corresponden en su mayoría a fragmentos de huesos largos; también se halló un fragmento de vértebra lumbar con marcas de corte y quemado. En el sitio EP se identificó un fragmento de cráneo y una falange de pinnípedo y escasas vértebras de peces. 


\begin{tabular}{|c|c|c|c|c|}
\hline \multicolumn{3}{|c|}{ Variables } & La Escondida & El Pajonal \\
\hline \multicolumn{3}{|c|}{${ }^{14} \mathrm{C}$ años AP } & $920 \pm 50($ LP- 3359) & - \\
\hline \multicolumn{3}{|c|}{ Años cal AP (2 ) } & $916-687$ & - \\
\hline \multicolumn{3}{|c|}{ Nacella magellanica } & $N R=264$ & $N R=178$ \\
\hline \multicolumn{3}{|c|}{ Mytilus edulis } & $N R=0$ & $N R=1$ \\
\hline \multirow{4}{*}{ 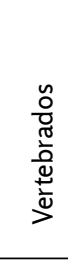 } & \multicolumn{2}{|l|}{$\begin{array}{l}\text { Pinnípedos } \\
\text { (F. Otariidae) }\end{array}$} & $\mathrm{NISP}=0$ & $\mathrm{NISP}=2$ \\
\hline & \multicolumn{2}{|l|}{$\begin{array}{l}\text { Guanacos } \\
\text { (Lama guanicoe) }\end{array}$} & $\mathrm{NISP}=10$ & $\mathrm{NISP}=0$ \\
\hline & \multicolumn{2}{|l|}{ Peces } & $\mathrm{NISP}=0$ & $\mathrm{NISP}=3$ \\
\hline & \multicolumn{2}{|l|}{ Indet. } & $\mathrm{NISP}=23$ & $\mathrm{NISP}=8$ \\
\hline \multirow{6}{*}{ 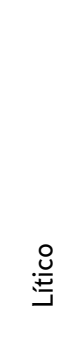 } & $\begin{array}{l}\text { Formas base } \\
\text { potenciales }\end{array}$ & $\begin{array}{l}\text { Lascas } \\
\text { Láminas }\end{array}$ & $\begin{array}{c}21(7,8 \%) \\
0\end{array}$ & $\begin{array}{c}23(24,2 \%) \\
3(3,1 \%)\end{array}$ \\
\hline & \multirow{4}{*}{ Desechos de talla } & Lascas chicas & $120(44,8 \%)$ & $22(23,1 \%)$ \\
\hline & & Microlascas & $33(12,3 \%)$ & $7(7,4 \%)$ \\
\hline & & Esquirlas & $72(26,9 \%)$ & $26(27,4 \%)$ \\
\hline & & Desechos & $22(8,2 \%)$ & $13(13,7 \%)$ \\
\hline & \multicolumn{2}{|l|}{ Núcleos } & o & $1(1 \%)$ \\
\hline
\end{tabular}

Tabla 2. Datación radiocarbónica, número de restos (NR) de moluscos, número de especímenes óseos identificados por taxón (NISP) y frecuencia de artefactos líticos en los sitios LE y EP.

El conjunto lítico consta de 95 artefactos en EP y 268 en LE (Tabla 2). En ambos sitios predominan los desechos de talla de tamaño muy chico (menor a $20 \mathrm{~mm} ; 83,2 \%, \mathrm{n}$ $=79$ en EP y $96 \%, n=257$ en LE) y no se hallaron instrumentos formatizados. En cuanto a las materias primas líticas en el sitio LE predomina la calcedonia (90\%), y le siguen la andesita $(3,7 \%)$, la riolita (3\%), la cuarcita $(1,8 \%)$ y el basalto $(1,5 \%)$. Por su parte, en el sitio EP predomina la calcedonia (59\%) y en menor porcentaje el basalto (21\%), la riolita $(8,4 \%)$, la cuarcita $(5,3 \%)$, la andesita $(3,1 \%)$ y el pórfido $(3,1 \%)$.

\section{Discusión y consideraciones finales}

Durante las tareas de campo se reconoció la presencia de materiales arqueológicos en torno a la totalidad de los cuerpos lagunares prospectados. En las lagunas más cercanas al litoral marítimo (hasta aproximadamente $3 \mathrm{~km}$ de distancia desde el mar; Figura 1) la densidad de sitios así como de los materiales que los componen es mayor que en las ubicadas hacia el interior. Los sitios registrados en las lagunas Los Médanos, Laguna Seca 1, Laguna La Llave y Salina Pancho corresponden a acumulaciones plano extendidas de restos líticos, óseos faunísticos y malacológicos $(70,5 \%, \mathrm{n}=67$ densidad baja y $29,5 \%, n=28$ densidad media; Figura 3B). En tanto que los sitios en las lagunas La Escondida, Shark y Laguna Seca 2 y 3 están formados por densidades bajas de materiales distribuidos de manera dispersa (Figuras 1 y $3 \mathrm{~B}$ ).

A partir de la localización de los sitios se pudo observar que estos se encuentran principalmente en las márgenes este y sur de los cuerpos lagunares (Figura 1). Dicha ubicación coincide con la presencia de mantos o plumas eólicas como consecuencia de la acumulación de sedimentos finos, influenciada principalmente por los vientos predominantes del oeste. El emplazamiento de los sitios principalmente en las márgenes sur y este de las lagunas podría relacionarse con la elección de los sustratos eólicos para el asentamiento, así como con su cercanía relativa al litoral atlántico. 
En los conjuntos arqueomalacológicos identificados durante las prospecciones así como en los sitios excavados, casi la totalidad de los exoesqueletos de moluscos corresponden a la especie Nacella magellanica. A partir de la Figura 3B se observa que existe un decrecimiento monotónico en sentido este-oeste en la abundancia de exoesqueletos calcáreos a medida que aumenta la distancia desde la línea de costa marina. Este patrón de disminución gradual en la abundancia de los exoesqueletos de moluscos se relacionaría con la existencia de mecanismos directos de aprovisionamiento (Renfrew, 1977) de estos recursos en el litoral marítimo. Se interpreta que los ejemplares de Nacella magellanica podrían haber sido recolectados en las plataformas de abrasión rocosas en los intermareales más cercanos ubicados sobre el frente atlántico, en los cuales se identificó que esta especie predomina ampliamente por sobre otros moluscos (Hammond y Zilio, 2016a). Así, sería posible calcular la distancia mínima de acarreo de los moluscos desde las plataformas de abrasión ubicadas en el litoral atlántico hasta las lagunas.

Los análisis biométricos realizados sobre los exoesqueletos completos de Nacella magellanica permitieron reconocer para LE las medidas de tamaño promedio más grandes registradas hasta el momento en la CNSC. Los tamaños obtenidos en EP también se ubican entre los más altos para el área de estudio (Hammond y Zilio, 2016b). Las medidas biométricas que se conocen para la CNSC provienen de exoesqueletos de moluscos recuperados en concheros de la franja de costa (Hammond y Zilio, 2016b), de esta manera, surge el interrogante que plantea si el tamaño promedio de las conchas de LE y EP, en comparación a aquellas registradas en concheros cercanos al litoral marítimo, podría deberse a una selección de los ejemplares por tamaño, teniendo en cuenta que las mismas se trasladaron hasta el sitio desde una distancia mínima de 5 $\mathrm{km}$ desde la costa. Como plantea Waselkov (1987), el costo de transportar a grandes distancias los recursos malacológicos es alto, debido a que la proporción de carne comestible en relación al peso total del molusco es generalmente baja. A partir de los resultados generados en este trabajo consideramos que se habrían seleccionado individualmente y trasportado hacia las lagunas los ejemplares de mayor tamaño y contenido de carne con el fin de obtener el máximo de alimento en cada traslado. Sin embargo, sería necesario ampliar la muestra de exoesqueletos de moluscos procedentes de sitios en lagunas a fin de analizar si esta tendencia inicial se continúa.

En los dos sondeos estratigráficos se reconoció la presencia de una variedad de taxones de vertebrados de procedencia tanto terrestre como marina (Tabla 2). El alto nivel de fragmentación observado en los restos óseos de fauna de gran tamaño (pinnípedos y guanaco), característico de conjuntos generados como resultado de las actividades de consumo y descarte, podría ser indicativo de las etapas finales del procesamiento que se desarrollan en el ámbito de la unidad de consumo (De Nigris, 2004).

A partir del análisis de los materiales artefactuales recuperados en las excavaciones se interpreta que en los sitios se llevaron a cabo principalmente tareas de talla, retalla y mantenimiento de instrumentos líticos. En los conjuntos se destaca la presencia de lascas, esquirlas, microlascas y lascas chicas (con módulos de tamaño chico y muy chico), productos de tareas de formatización final, reactivación de filos y rebaje bifacial de artefactos. Además se identificó una gran variedad de materias primas líticas. En el sitio EP se registró la técnica de talla bipolar sobre materias primas de disponibilidad local como los rodados pequeños de basalto negro. Dicha técnica constituye una estrategia tecnológica eficiente para la obtención de lascas a partir de los rodados costeros y para maximizar el aprovechamiento de las materias primas.

Las evidencias arqueológicas registradas en el sector estudiado permiten afirmar que los grupos humanos desarrollaron diferentes actividades cotidianas en espacios inmediatos a los cuerpos lagunares tales como el procesamiento y consumo de recursos, 
la manufactura y mantenimiento de instrumentos líticos, entre otras. En general las bajas densidades que conforman los conjuntos arqueológicos identificados en torno a las lagunas sugerirían que las ocupaciones de este espacio habrían sido por cortos períodos de tiempo.

La datación radiocarbónica realizada en LE permitió ubicar cronológicamente al sitio en el Holoceno tardío final (Tabla 2). Es necesario realizar nuevas dataciones en otros contextos arqueológicos a fin conocer el lapso temporal de uso de estos espacios lagunares.

La alta frecuencia de sitios arqueológicos en el sector analizado contrasta notablemente con la ausencia de estos en la franja de costa atlántica adyacente (Castro et al., 2003). Se plantea como hipótesis que los espacios lagunares estudiados en este trabajo habrían sido más atractivos para el asentamiento de las poblaciones humanas que la franja de costa marítima más cercana. Los cuerpos lagunares podrían haber funcionado como atractores o factores de localización debido principalmente a la disponibilidad de agua dulce, que en ciertos momentos del año o luego de períodos de lluvias se habría acumulado en las lagunas. De la misma manera, estos espacios habrían sido atractivos para diversas especies faunísticas y vegetales en los momentos en que poseían agua (aún salobre), lo que podría haber influenciado en la selección de este sector por parte de los grupos humanos debido a la concentración de recursos. Por otro lado, los sustratos característicos de las márgenes de los cuerpos lagunares están representados por mantos eólicos y sedimentos finos depositados por acción del viento y el agua, en tanto que las amplias playas de progradación del litoral atlántico están constituidas exclusivamente por rodados litorales. Este hecho establece la presencia de distintos sustratos potenciales de asentamiento humano en ambos sectores. No obstante, los espacios elegidos para el asentamiento correspondieron a las márgenes de las lagunas, en tanto que en el sector de litoral marítimo se realizaron actividades de recolección y aprovisionamiento de moluscos y vertebrados marinos, además de rodados litorales, recursos que se transportaron posteriormente hacia los cuerpos lagunares.

Las evidencias analizadas en este trabajo permiten postular el uso diferencial del sector lagunar y la franja marítima adyacente, a partir del cual los grupos cazadores recolectores habrían implementado estrategias de circulación basadas en la complementariedad del uso de los espacios teniendo en cuenta la estructura de los recursos (agua dulce, vertebrados, invertebrados, materias primas líticas, vegetales, entre otros) y las características de los sustratos de ocupación disponibles.

\section{Agradecimientos}

Al señor Adrián Fernández de la Estancia El Pajonal. Al Dr. Enrique Fucks por la provisión de información geomorfológica. A los dos evaluadores anónimos y a los editores de la revista quienes mediante sus comentarios y sugerencias permitieron mejorar el artículo. Este trabajo se realizó en el marco del proyecto "Estudios regionales en el eje Deseado (cuencas media e inferior) para definir los rangos de acción de grupos cazadores recolectores costeros en el marco de la ocupación humana del Holoceno de Patagonia" (11/N 739, UNLP) dirigido por la Dra. A. Castro. A la Universidad Nacional de la Plata y al Consejo Nacional de Investigaciones Científicas y Técnicas (Becas Postdoctorales de los autores). 


\section{Q Referencias citadas}

"Alonso, R. N. (2006). Ambientes evaporíticos continentales de Argentina. Instituto Superior de Correlación Geológica, 21, 155-170.

"Ambrústolo, P. (2011). Estudio de las estrategias de aprovisionamiento y utilización de los recursos líticos por los grupos cazadores-recolectores en la costa norte de Santa Cruz. (Tesis Doctoral inédita), Universidad Nacional de La Plata, Argentina.

» Aschero, C. (1975). Ensayo para una clasificación morfológica de artefactos líticos aplicada a estudios tipológicos comparativos. Informe al CONICET. Manuscrito inédito.

» Bronk Ramsey, C. y Lee, S. (2013). Recent and planned developments of the program OxCal. Radiocarbon, 55, 3-4.

"Castro, A. S. (1994). El análisis funcional por medio del estudio microscópico de huellas de uso. (Tesis Doctoral inédita), Universidad Nacional de La Plata, Argentina.

" Castro, A. S. y Moreno, J. E. (2000). Noticia sobre enterratorios humanos en la costa norte de Santa Cruz-Patagonia-Argentina. Anales del Instituto de la Patagonia, 28, 225-232.

»Castro, A., Moreno, J. E., Andolfo, M. A., Giménez, R., Peña, C., Mazzitelli, L., Zubimendi, M. A. y Ambrústolo, P. (2003). Análisis distribucionales en la costa de Santa Cruz (Patagonia Argentina): alcances y resultados. Magallania, 31, 69-94.

"Castro, A. S., Moreno, J. E., Andolfo, M. y Zubimendi. M. A. (2001). Distribución espacial de sitios en la localidad de Punta Medanosa, Santa Cruz (Argentina). Relaciones de la Sociedad Argentina de Antropología, XXVI, 303-322.

" Codignotto, J. O. (1997). Geomorfología y dinámica costera. El Mar Argentino y sus Recursos Pesqueros, 1, 89-105.

"De Nigris, M. E. (2004). El Consumo en Grupos Cazadores Recolectores. Un Ejemplo Zooarqueológico de Patagonia Meridional. Buenos Aires: Sociedad Argentina de Antropología.

" Foley, R. (1981). A model of regional archaeological structure. Proceedings of the Prehistoric Society 47, 1-17.

" Hammond, H. (2015). Sitios concheros en la costa norte de Santa Cruz: su estructura arqueológica y variabilidad espacial en cazadores recolectores patagónicos. (Tesis Doctoral inédita), Universidad Nacional de La Plata, Argentina.

» Hammond, H. y Zilio, L. (2016a). Experimentación del proceso de recolección de moluscos marinos en la costa patagónica: análisis de la especie Nacella magellanica y sus implicancias en la interpretación de muestras arqueomalacológicas. Comechingonia, 20(2), $265-290$.

» Hammond, H. y Zilio, L. (2016b). Cambios en el tamaño de exoesqueletos calcáreos de moluscos durante el holoceno tardío: arqueomalacología de concheros en la costa norte de Santa Cruz, Patagonia argentina. Arqueología Iberoamericana, 32, 17-24.

» Hogg, A. G., Hua, Q., Blackwell, P. G., Niu, M., Buck, C. E., Guilderson, T. P., Heaton, T. J., Palmer, T. G., Reimer, P. J., Reimer, R. W., Turney, C. S. M. y Zimmerman, S. R. H. (2013). SHCal ${ }_{13}$ Southern Hemisphere calibration, 0-50,000 cal yr BP. Radiocarbon, 55(4), 18891903.

》 Lyman, R. L. (1994). Vertebrate Taphonomy. Nueva York: Academic Press. 
" Moreno Jiménez, A. (2005). Modelización cartográfica de densidades mediante estimadores Kernel. Treballs de la Societat Catalana de Geografía, 30, 155-170.

»Pedoja, K., Regard, V., Husson, L., Martinod, J., Guillaume, B., Fucks, E., Iglesias, M. y Weill, P. (2011). Uplift of Quaternary shorelines in eastern Patagonia: Darwin revisited. Geomorphology, 127(3), 121-142.

»Renfrew, C. (1977). Alternate Models for Exchange and Spatial Distribution. En T. K. Earle (Ed.), Exchange Systems in Prehistory (pp. 71-89). Nueva York: Academic Press.

"Waselkov, G. A. (1987). Shellfish gathering and shell midden archaeology. En M. B. Schiffer (Ed.), Advances in Archaeological Method and Theory (Volume 10) (pp. 93-210). Orlando: Academic Press.

»Zubimendi, M. A. (2012). La variabilidad del registro arqueomalacológico en la costa norte de Santa Cruz (Patagonia argentina): resultados exploratorios a partir de estudios estratigráficos. Intersecciones en Antropología, 13(2), 359-375.

»Zubimendi, M. A., Castro, A. S. y Moreno, E. J. (2004). Una aproximación hacia la definición de modelos de uso de la costa norte de Santa Cruz. Magallania, 32, 209-220. 